\title{
Bioindicadores ambientais: insetos como um instrumento desta avaliação
}

\author{
Marco Antonio de Oliveira1, Cliver Fernandes Farder Gomes ${ }^{2}$, Evaldo Martins Pires ${ }^{3}$, \\ Cidália Gabriela Santos Marinho ${ }^{4}$, Terezinha Maria Castro Della Lucia ${ }^{5}$
}

http://dx.doi.org/10.1590/0034-737X201461000005

\begin{abstract}
RESUMO
Atividades agrícolas, florestais e do setor imobiliário vêm, ao longo do tempo, modificando ambientes ecologicamente estruturados. As consequências são drásticas e, por isso, faz-se necessária a busca por ferramentas e instrumentos para diagnosticar e monitorar a diversidade biológica desses locais. Os insetos têm-se destacado como potenciais organismos bioindicadores e isso se deve ao fato de apresentarem grande capacidade perceptiva, no que se refere a alterações do meio ambiente, principalmente por seu apurado sistema sensorial, que lhes permite qualificar condições ambientais em determinadas situações e, ainda, quantificar danos causados ao meio. Um dos problemas que podem ser associados a este setor é a falta de padronização e definição de protocolos de coleta e avaliação da biodiversidade, para que esses resultados possam ser analisados e extrapolados para diferentes ambientes antropizados. Neste trabalho, é contextualizada a importância da entomofauna como bioindicadora, em ecossistemas.
\end{abstract}

Palavras-chave: Biodiversidade, bioindicadores, impacto ambiental, insetos.

\section{ABSTRACT}

\section{Environmental bioindicators: insects as a tool for biodiversity monitoring}

Agricultural, forestry and real estate activities have been modifying ecologically structured environments. The consequences of these activities are drastic and so it is necessary to search for tools to diagnose and monitor biological diversity of these environments. Insects have been identified as potential biomarkers because of their great ability to perceive changes in the environment, mainly due to their acute sensory system that allows them to evaluate the environmental conditions in certain situations, as well as to quantify damages to the environment. However, there is a lack of standardization and definition of protocols for the collection and assessment of biodiversity so that these results can be analyzed and extrapolated to different anthropogenic environments. This study contextualized the importance of the insect fauna as bioindicators in different ecosystems

Key words: biodiversity, biomarkers, environmental impact, insects.

Recebido para publicação em 15/05/2014 e aprovado em 15/10/2014.

${ }^{1}$ Engenheiro Florestal, Doutor. Instituto de Ciências Biológicas e da Saúde, Universidade Federal de Viçosa, Campus UFV Florestal, Rodovia LMG 818, km 06, 35690-000, Florestal, Minas Gerais, Brazil. marco.oliveira@ufv.br (autor para correspondência).

2. Graduando em Ciências Biológicas. Universidade Federal de Viçosa, Campus UFV Florestal, Rodovia LMG 818, km 06, 35690-000, Florestal, Minas Gerais, Brazil. clivermetal@hotmail.com

3. Biólogo, Doutor. Universidade Federal de Mato Grosso, Campus Sinop, Avenida Alexandre Ferronato, 1200, 78557-267, Sinop, Mato Grosso, Brasil. evaldo.pires@gmail.com 4. Engenheira-Agrônoma, Doutora. Universidade Federal de São João del-Rei, Campus Sete Lagoas, Rodovia MG 424, Km 47, 35701-970, Sete Lagoas, Minas Gerais, Brasil. gabriela@ufsj.edu.br

5. Bióloga, Doutora. Instituto de Ciências Biológicas e da Saúde, Universidade Federal de Viçosa, Campus Viçosa, Avenida Peter Henry Rolfs, 36570-000, Viçosa, Minas Gerais, Brasil. tdlucia@ufv.brcidade. 


\section{INTRODUÇÃO}

O homem, ao longo de sua existência, vem provocando alterações no ambiente, transformando paisagens estruturadas em ambientes simplificados e, consequentemente, reduzindo a biodiversidade. Essa transformação pode, ainda, levar à exclusão de espécies-chave dos ecossistemas, afetando a flora, a fauna, as relações ecológicas entre os organismos e prejudicando a qualidade de vida no planeta (Didham, 1997).

Dentre os invertebrados, vários organismos têm sido utilizados como bioindicadores dessas transformações (Brown, 1997; Edge, 2005; Uys et al., 2006; Engelbrecht, 2010; McGeoch et al., 2011). Esses organismos podem ser agrupados em três categorias principais: os indicadores ambientais; os indicadores ecológicos e os indicadores de biodiversidade (Hellawell, 1986; Paoletti \& Bressan, 1996; Meffe \& Carroll, 1997; Flather et al., 1997; Gaston \& Blackburn, 1995).

Os "indicadores de biodiversidade" têm-se destacado, principalmente, pelo crescente número de trabalhos envolvendo a escolha de áreas prioritárias para conservação (Margules et al., 1988). Uma questão que pode influenciar negativamente nesses estudos é a falta de especialistas na área de taxonomia, fato que pode limitar o conhecimento da diversidade e da distribuição dos invertebrados nos ecossistemas (Vane-Wright et al., 1994).

Atualmente, vários países estão desenvolvendo programas de monitoramento dos ambientes, utilizando bioindicadores, já que muitos empreendimentos exigem informações sobre a qualidade do ambiente para que recursos financeiros sejam liberados (Cohen, 2003; Niemi \& McDonald, 2004; McGeoch et al., 2011; Soares-Filho et al., 2012; Gorenflo et al., 2012). A efetivação do uso de bioindicadores nas atividades de monitoramento requer, ainda, estudos, além de uma política e legislação pertinente para a escolha desses organismos (Hellawell, 1991).

A primeira referência de bioindicadores, feita por Platão, citou os impactos da atividade humana sobre a produção de frutas (Rapport, 1992). Morrison (1986), revisando os trabalhos de Clements (1920), observou que os primeiros conceitos de bioindicadores para comunidades de plantas e animais dataram do século XVI; e que o uso de organismos com esta finalidade tem sido desenvolvido em diversos setores. Por exemplo, o uso de pássaros, para monitorar as condições do ar em minas subterrâneas (Burrell \& Siebert, 1916); o de bioindicadores aquáticos, na qualidade da água (Winner et al., 1980; Hellawell, 1986; Rapport, 1992; Rosenberg \& Resh, 1993) e, mais recentemente, o uso de invertebrados como bioindicadores terrestres (Dobson, 2005; Mendelik et al., 2005; Gardner et al., 2008).

\section{INSETOS COMO POTENCIAIS BIOINDICADORES DE QUALIDADE AMBIENTAL}

Dentre os insetos com potencial para uso em programas de monitoramento ambiental, as principais espécies pertencem às ordens Coleoptera, Diptera, Hemiptera, Hymenoptera, Lepidoptera e Orthoptera (Brown, 1997). Esses insetos destacam-se pelo papel que desempenham no ecossistema: a ciclagem de nutrientes, a decomposição, a produtividade secundária, a polinização, o fluxo de energia, a predação, a dispersão de sementes, a regulação das populações de plantas e de outros organismos (Price, 1984).

A ordem Coleoptera é considerada a mais diversa entre os insetos, com aproximadamente 300.000 espécies descritas. Esse grupo sobressai, também, pela abundância nos mais diferentes ecossistemas e atua em vários níveis tróficos, o que pode classificá-los como pragas agrícolas, polinizadores, dispersores de sementes, predadores e decompositores (Triplehorn \& Jonnson, 2011). Apresentam grande diversidade de comportamentos e de funções nos ecossistemas. São sensíveis às mudanças ambientais, que afetam diretamente a riqueza, a distribuição, a abundância e até a estrutura de suas guildas. As mudanças nos padrões de comportamento e na abundância sazonal dos insetos têm sido utilizadas como ferramenta para explicar os distúrbios ambientais, em várias partes do mundo (Pearce \& Venier, 2006; Freitas et al., 2006; Nichols et al., 2007).

Algumas famílias de besouros, como Carabidae, Cerambycidae, Chrysomelidae, Curculionidae e Elateridae, vêm sendo referenciadas pelos bons resultados como bioindicadoras e, ainda, por apresentarem facilidade de coleta e, nas relações com o ecossistema, alta fidelidade ecológica, diversificação taxonômica (Brown Junior, 1996), e por serem rapidamente afetadas quando ocorre algum tipo de modificação ou fragmentação de ambiente (Didham, 1998).

Otavo et al. (2013) avaliaram o efeito do gradiente de perturbação antrópica, na Amazônia, sobre a superfamília Scarabaeoidea, e concluíram que esses besouros não podem ser utilizados como único elemento bioindicador de impacto, por causa das grandes diferenças que existem em seus componentes (famílias, subfamílias, gêneros e espécies) e das diferentes respostas que apresentam em áreas impactadas. Esses mesmos autores observaram, ainda, que a riqueza e abundância das famílias Dynastidae e Scarabaeidae aumentaram com a intensidade dos impactos sofridos pelo ambiente. Nesse mesmo estudo, foi observado, também, que besouros das famílias Hybosoridae e Melolonthidae re- 
duzem a sua ocorrência à medida que os impactos aumentam. Isso demonstra a complexidade para afirmarse o uso de um inseto ou grupo como bom bioindicador, pois, as relações do inseto com o ambiente podem sofrer alterações e respostas diferenciadas, dificultando uma padronização das afirmativas obtidas nos diferentes trabalhos.

Louzada \& Lopes (1997), avaliando áreas fragmentadas, cercadas por ambientes diversificados, concluíram que o isolamento dos representantes de Scarabaeidae pode levar a endocruzamentos, e até, à extinção das populações locais. Outro fator de redução das espécies de Scarabaeidade é o uso do fogo na vegetação de restinga, no litoral do Espírito Santo (Louzada \& Shifler, 1996). Esses resultados comprovam que o desmatamento e as práticas agrícolas e industriais têm levado à eliminação de algumas espécies de besouros escarabeídos "rola bosta" na floresta tropical.

Estudos de Hutcheson (1990) e Marinoni \& Dutra (1997) demonstraram que coleópteros herbívoros predominam em áreas degradadas em início de regeneração, enquanto os grupos de detritívoros e fungívoros apresentaram maior ocorrência em áreas conservadas. Esses insetos também são sensíveis às mudanças nos índices de precipitação de determinadas regiões, sendo mais abundantes nos meses de chuva (Louzada \& Lopes, 1997; Freitas et al., 2006).

Outra ordem de interesse na bioindicação é a dos Hymenoptera, representados pelas abelhas, formigas e vespas. Compreendem cerca de 115.000 espécies descritas, várias com importância comprovada no ambiente (LaSalle \& Gauld, 1993).

As vespas e abelhas solitárias, por exemplo, são importantes pelas interações que mantêm com outros grupos de organismos (parasitismo, predação, polinização, dispersão) e também porque as variações espaciais e temporais de sua abundância e riqueza têm demonstrado correlações importantes com as alterações na estrutura do ambiente e com a diversidade de outros organismos (Morato \& Campos, 2000; Morato 2001; Martins \& Antonini, 2002; Barbieri Junior et al., 2012). Esse comportamento também foi observado, por Brown e Albrecht (2001), em abelhas sem ferrão do gênero Melipona, em floresta tropical em Rondônia, as quais são sensíveis ao desmatamento. De modo geral, os insetos deste grupo são sensíveis às mudanças ecológicas, principalmente às referentes à estrutura, à composição da vegetação e, também, aos resíduos de moléculas de inseticidas, de fungicidas e de poluentes presentes nas plantas. Essa sensibilidade tem sido utilizada como ferramenta para avaliar a qualidade dos ecossistemas, em áreas de mineração, de agricultura, ou urbanas (Olivier et al., 2012).
As formigas, por sua vez, têm seu destaque neste contexto por apresentarem: 1) ampla distribuição geográfica; 2) abundância local elevada e alta riqueza de espécies; 3) resposta rápida ao stress do meio; 4) facilidade de amostragem e de identificação, quando comparadas com as de outros organismos (Majer, 1983). Elas estão distribuídas em 322 gêneros, 16 subfamílias e 12.955 espécies (Bolton, 2014), sendo que 119 gêneros estão na região neotropical, pertencentes a oito subfamílias, com aproximadamente 3.100 espécies descritas (Fernández \& Ospina, 2003). Atuam nos ecossistemas como predadoras, herbívoras, saprófagas, polinizadoras, dispersoras de sementes, afetando diretamente a estrutura e composição da vegetação (Hölldobler \& Wilson, 1990; Folgarait, 1998; Della Lucia, 2011). Algumas espécies são problemas como pragas agrícolas e florestais (Oliveira et al., 2011) ou como pragas urbanas (Chacón de Ulloa, 2003).

A família Formicidae constitui um dos grupos de insetos mais estudados e analisados. As informações hoje disponíveis na literatura têm permitido conclusões interessantes, comprovando a importância desses insetos no contexto da conservação. Underwood \& Fisher (2006), analisando 60 publicações de várias partes do mundo, com diferentes metodologias utilizadas para coletar formigas, reforçam sua importância no acompanhamento das mudanças nos ecossistemas. Contudo, mencionam a falta de estudos aplicados e técnicas de gestão para o uso efetivo das informações adquiridas. Neste contexto, Alonso \& Agosti (2000) publicaram um protocolo de coleta de formigas no intuito de facilitar futuras comparações dos resultados obtidos nos diferentes ecossistemas.

Segundo Majer (1983), vários trabalhos têm encontrado correlação da riqueza total de espécies de formigas com a riqueza de alguns gêneros ou grupos de formigas, ou com a riqueza de taxa superiores. Andersen (1997b) encontrou gêneros de formigas cuja riqueza local apresentou uma forte correlação com a riqueza total de formigas, o que pode ser também avaliado regionalmente ou, mesmo em habitats. Andersen \& Sparling (1997) determinaram a riqueza de espécies de formigas associadas a outros grupos e encontraram correlação positiva entre riqueza de espécies de formigas e a biomassa microbiana dos solos, em áreas de mineração, impactadas, na Austrália. Oliver \& Beattie (1996) sugeriram que a riqueza de formigas pode ser útil como indicador da diversidade beta de coleópteros e aranhas e que, em alguns casos, informações sobre a fauna de formigas, somadas às informações de famílias de coleópteros, são úteis para avaliar a biodiversidade de invertebrados em geral. Vários são os estudos que relatam a importância das formigas como bioindicadoras (Silva \& Brandão, 
1999; Ribas et al., 2012). Elas foram estudadas em diversos ambientes, como: áreas de reabilitação de minas, na Austrália (Majer \& Nichols, 1998), na África do Sul (Majer \& Kock, 1992) e no Brasil (Oliveira \& Della Lucia, 1992; Majer, 1996; Santos et al., 2008); em áreas com metais pesados (Ribas et al., 2012); em áreas de cultivo agrícola (Peck et al., 1998); em avaliação de distúrbio em florestas tropicais (Marinho et al., 2002; Delabie et al., 2006; Leal et al., 2012; Miranda et al.,2013) e, ainda, em áreas cultivadas tratadas com inseticidas (Couto et al., 2010; Pereira et al. 2010) e herbicidas (Barros et al., 2010).

Geralmente, os estudos têm resultado em maior riqueza de espécies de formigas, associada a maior riqueza de espécies de plantas e, também, ao tempo decorrido do processo de recomposição das áreas que sofreram algum tipo de distúrbio. Isso corrobora a hipótese de que os ambientes mais complexos podem oferecer melhores condições para a manutenção e sobrevivência de maior número de espécies da mirmecofauna (Andersen, 1986; King et al., 1998; Oliveira et al., 1995; Vasconcelos, 2008; Oliveira et al., 2009). Fatores como a heterogeneidade da serapilheira (Brühl et al., 1998), as condições climáticas (Levings, 1983), a complexidade estrutural e a heterogeneidade da vegetação podem modificar as condições ambientais e, consequentemente, a diversidade da fauna de formigas (Oliveira et al., 2011).

Trabalhos realizados na Austrália e no Brasil (Majer, 1983; Alonso, 2000) propuseram o uso de grupos funcionais de formigas, pois, um conjunto de espécies de formigas quando exposto a diferentes condições ambientais, apresenta padrões de resposta previsíveis, podendo ser agrupadas. Quando analisadas conjuntamente com as propriedades ambientais, podem fornecer importantes respostas sobre a qualidade do ambiente avaliado. Características como preferência de habitat, microclima, dominância, estratégias de forrageamento, nível de tolerância às perturbações ambientais, interações comportamentais, dentre outras, podem ser utilizadas para definir os grupos funcionais (Andersen, 1997a; Silvestre et al., 2003). A aplicação dos modelos de grupos funcionais tem fortalecido o uso de formigas como bioindicadores, mostrando a capacidade de comparação de resultados entre regiões e diferentes ecossistemas.

A ordem Diptera é representada por cerca de 10.000 gêneros, 150 famílias com uma estimativa de 150.000 espécies descritas (Pape et al., 2009). Estão distribuídas em todos os continentes, incluindo a Antártica, com alta capacidade de colonização, principalmente no estádio larval, em ambientes aquáticos, sendo o mar aberto o único ambiente inexplorado por esses in- setos (Courtney \& Merritt, 2008). São conhecidos como moscas, mosquitos e mutucas, mais lembrados pelo interesse médico-veterinário. Exercem, entretanto, importantes funções como decompositores, polinizadores e controladores biológicos, fato que reforça sua importância (Triplehorn \& Jonsson, 2011).

Portanto, conhecer as espécies de dípteros e analisar sua ocorrência em diferentes ambientes é relevante. Por exemplo, as espécies Mesembrinella bellardiana e Mesembrinella peregrina (Calliphoridae) são encontradas apenas em locais preservados da mata atlântica. Gadelha et al. (2009) corroboram esse fato e sugerem que as espécies desse gênero podem auxiliar na averiguação de prováveis impactos ambientais. Assembleias de drosofilídeos também têm sido usadas para avaliar os distúrbios humanos na região do cerrado brasileiro (Mata et al., 2008). Outro exemplo são as famílias de dípteros importantes no processo de decomposição da matéria orgânica do solo (Frouz, 1999), destacando-se Chironomidae, Syrphidade, Cecidomyiidae e Sciaridae. Essas duas últimas famílias são muito sensíveis aos agrotóxicos e a fertilizantes no solo (Buchs et al., 2003). Os representantes de Syrphidae não têm sido encontrados em áreas agrícolas ou áreas degradadas, em função da alta exigência de serapilheira e umidade para sua sobrevivência (Frouz, 1999); portanto, são bons bioindicadores.

Os insetos da ordem Lepidoptera também têm sido considerados grupos-chave como bioindicadores, pois atuam nos ecossistemas florestais e agrícolas como desfolhadores, decompositores, presas e hospedeiros de carnívoros. São influenciados pela dinâmica populacional das plantas e, também, pelas relações predador-presa, respondendo rapidamente a qualquer mudança que possa ocorrer no ambiente (Kremen, 1992; Brown Junior, 1977; 1996; Hammond \& Miller, 1998). O desmatamento tem sido um dos fatores que afetam a riqueza, a abundância e a uniformidade da fauna de lepidópteros em florestas tropicais (Hill et al., 1995; Spitzer et al., 1997). Entretanto, o hábito herbívoro desses insetos leva a comportamentos e respostas diferenciados, muitas vezes dificultando o isolamento dos fatores responsáveis pelos distúrbios (Wood \& Gillman, 1998; Hammer et al., 1997).

Existe também um grupo de organismos, chamados de macroinvertebrados bentônicos, que têm importante papel como bioindicadores, por apresentarem grande tolerância e amplo espectro de respostas frente a diferentes níveis de contaminação do ambiente aquático (Weston \& Lydy, 2014). Segundo Resh \& Jackson (1993), eles podem ser considerados bons bioindicadores, por apresentarem ciclos de vida geralmente longos, tamanho de corpo relativamente grande, que facili- 
ta a amostragem, alta diversidade e técnicas de amostragem padronizadas e de baixo custo.

Dentro desse grupo merecem destaque os Odonata; os Diptera (Chironomidae; Ceratopogonidae, Simuliidae (águas limpas) e os Tipulidade (águas contaminadas); os Coleoptera, os Trichoptera (águas correntes, limpas e oxigenadas), os Ephemeroptera e os Hemiptera. Cada ordem tem, em seus representantes, características importantes que podem ser utilizadas nesses estudos (Merrit et al., 2008).

Segundo Callisto et al. (2001), quando a identificação taxonômica específica não for possível para a caracterização das comunidades bentônicas e dos seus principais processos, tem-se trabalhado com outras unidades ecológicas, como as categorias de grupos tróficos funcionais. Esta classificação considera o hábito alimentar dos organismos e a utilização dos recursos tróficos disponíveis, independentemente da identificação das espécies. Os protocolos de coleta em ambientes aquáticos já estão bem definidos para avaliar a qualidade das águas nos seus aspectos físicos, químicos e biológicos (Chernoff et al., 1998).

De modo geral, ambientes aquáticos conservados tendem a apresentar elevada diversidade de organismos, quando comparados com ambientes que sofreram algum tipo de impacto humano severo (Barbosa et al., 2001; Kemp et al., 2014). A exemplo disto, RizoPatron et al. (2013) usaram as comunidades de macroinvertebrados para avaliar a qualidade da água, em lavouras convencionais e orgânicas, de arroz irrigado, em Guanacaste, Costa Rica. Observaram que 76,5\% das espécies de macroinvertebrados coletados eram principalmente insetos das ordens Plecoptera, Trichoptera e Ephemeroptera, afetados por agrotóxicos utilizados para o combate de pragas. Alguns grupos de insetos aquáticos podem auxiliar na avaliação da poluição por metais pesados, conforme Numelin et al. (2007), em estudos realizados em áreas poluídas por metais. Foi encontrado que insetos da ordem Hemiptera (adultos da família Gerridae) apresentaram altas concentrações de ferro e manganês. Já as formas imaturas de Odonata apresentaram maior concentração de ferro e de cádmio. Norris \& Thoms (1999) também avaliaram o lançamento de esgoto ou de efluentes industriais sobre o comportamento desses grupos aquáticos. Corbi et al. (2011) observaram a presença de metais em predadores da família Belostomatidae (Hemiptera) e Libellulidae (Odonata), em áreas com cultivos de cana-de-açúcar, no Brasil, associada ao uso de fertilizantes nessa lavoura. Esses resultados demonstram o potencial de uso desses insetos para auxiliar em programas de monitoramento de metais pesados, nos diferentes ecossistemas.

\section{CONSIDERAÇÕES FINAIS}

A utilização de insetos como bioindicadores reforça uma linha de pesquisa que divulga a necessidade de cuidados com o ambiente. Isto porque, ao determinar a baixa ocorrência de espécies indicadoras, ou ainda a sua extinção por meio de estudos de levantamento de diversos grupos de insetos, como aqui apresentado, toma-se conhecimento de como as atividades agrícolas, florestais, do setor imobiliário, da mineração, dentre outras, têm prejudicado o funcionamento do meio em que vivemos. A despeito da vasta literatura acerca do tema, muito ainda falta ser descoberto e, por isso, informações precisas sobre avaliação da qualidade de habitats tornar-se-ão ferramenta de avaliação ambiental segura e confiável, a ponto de determinar quais medidas deveriam ser tomadas para o re-estabelecimento local. Para isto acontecer, é necessária a padronização de metodologias e protocolos de coleta, para que haja comparabilidade entre resultados obtidos em diferentes regiões e situações, utilizando-se as ferramentas computacionais e de modelagem hoje disponíveis. Isto permitirá que resultados obtidos de forma isolada e temporal possam ser reavaliados em outros momentos, dando condições do acompanhamento da evolução ou da recuperação do impacto sofrido.

\section{AGRADECIMENTOS}

Àqueles que nunca nos deixaram faltar a inspiração para apresentar o que a Natureza tem nos permitido elucidar.

\section{REFERÊNCIAS}

Alonso LE \& Agosti D (2000) Biodiversity studies, monitoring, and ants: An overview. In: Agosti E, Majer JD, Alonso LE \& Schultz TR (Eds.) Ants: standard methods for measuring and monitoring biodiversity. Washington, Smithsonian Institution Press. 18p.

Alonso LE (2000) Ants as indicators of diversity. In: Agosti D, Majer J, Alonso E \& Schultz TR (Eds.) Ants: standard methods for measuring and monitoring biodiversity. Washington, Smithsonian Institution Press. 280p.

Andersen AN \& Sparling GP (1997) Ants as indicators of restoration success: relationship with soil microbial biomass in the Australian seasonal tropics. Restoration Ecology, 5:109-114.

Andersen AN (1986) Diversity, seasonality and community organization of ants at adjacent heath and woodland sites in south-eastern Australia. Austral Journal Zoological, 34:53-64.

Andersen AN (1997a) Functional groups and patterns of organization in North american ant communities: a comparation with Australia. Journal of Biogeography, 24:433-460.

Andersen AN (1997b) Using Ants as bioindicators: Multiscale Issues in Ant Community Ecology. Conservation Ecology, 1:8.

Barbieri Junior CA \& Dias AMP (2012) Braconidae (Hymenoptera) fauna in native, degraded and restoration areas of the Vale do Paraíba, São Paulo state, Brazil. Brazilian Journal of Biology, 72:305-310. 
Barbosa FAR, Callisto M \& Galdean N (2001) The diversity of benthic macroinvertebrates as an indicator of water quality and ecosystem health: A case study for Brazil. Aquatic Ecosystem Health \& Management, 4:51-59.

Barros EC, Picanço MC, Pereira JP, Silva AA, Reis MR \& Castro Neto MD (2010) Formigas utilizadas como bioindicadoras da presença de herbicida e inseticida. In: $27^{\text {a }}$ Congresso Brasileiro da Ciência das Plantas Daninhas, Ribeirão Preto. Anais, Pantas daninhas. p.3272-3275.

Bolton B (2014) Taxonomic History. Disponível em: <http:// www.antweb.org/description.do?name=formicidae \&rank=family \&project=allantwebants $>$. Acessado em: 23 de Junho de 2014.

Brown JC \& Albrecht C (2001) The effect of tropical deforestation on stingless bees of the genus Melipona (Insecta: Hymenoptera: Apidae: Meliponini) in central Rondonia, Brazil. Journal of Biogeography, 28:623-634

Brown Junior KS (1977) Centros de evolução, refúgios quaternários e conservação de patrimônios genéticos na região neotropical: padrões de diferenciação em Ithomiinae (Lepidoptera: Nymphalidae). Acta Amazônica, 7:75-137.

Brown Junior KS (1996) The use of insects in the study, inventory, conservation and monitoring of biological diversity in Neotropica habitats, in relation to traditional land use systems. In: Ae SA, Hirowatari T, Ishii M \& Brower LP (Eds.) Decline and Conservation of Butterflies in Japan. Osaka, Lepidopterological Society of Japan. p.128-49.

Brown KS (1997) Diversity, disturbance, and sustainable use of Neotropical forests: insects as indicators for conservation monitoring. Journal of Insect Conservation, 1:25-42.

Brühl CA, Gunsalam G \& Linsenmair KE (1998) Stratification of ant (Hymenoptera, Formicidae) in a primary rain forest in Sabah, Borneo. Journal of Tropical Ecology, 14:285-297.

Buchs W, Harenberg A, Zimmerman J \& Weiss B (2003) Biodiversity, the ultimate agri-environmental indicator? Potential and limits for the application of faunistic elements as gradual indicators in agroecosystems. Agriculture Ecosystems and Environment, 98:99-123.

Burrell GA \& Siebert FM (1916) Gases found in coal mines. In: Burrell GA \& Siebert FM (Eds.) Publications on mine accidents and methods of mining. Washington, United States Government printing office. p.21-23.

Callisto M, Moretti M \& Goulart M (2001) Macroinvertebrados Bentônicos como Ferramenta para Avaliar a Saúde de Riachos. Revista Brasileira de Recursos Hidrícos, 6:71-82.

Chacón de Ulloa (2003) Hormigas urbanas. In: Fernández F (Eds.) Introducción a las hormigas de la región Neotropical. Bogotá, Instituto de Investigación de Recursos Biológicos Alexander Von Humbolt. p.351-359.

Chernoff B, Allonso A \& Hortega H (1998) Aqua-rap protocol Field Museum Chicago and Conservation International. Disponível em: <https://www.fieldmuseum.org/sites/default/files/ annual_report1998_0.pdf >. Acessado em: 27 de Junho de 2014.

Clements FE (1920) Palnt indicators: The relation of plant communities to process and practice. Washington, Carnegie Institute. 388p.

Cohen JE (2003) Human population: the next half century. Science, 302:1172-1175

Corbi JJ, Froehlich G, Trivinho-Strixino S \& Santos A (2011) Evaluating the use of predatory insects as bioindicators of metals contamination due to sugarcane cultivation in neotropical streams. Environmental Monitoring and Assessment, 177:545-554.

Courtney GW \& Merritt RW (2008) Larvae of aquatic Diptera. In: Merritt RW, Cummins KW \& Berg MB (Eds.) An Introduction to the Aquatic Insects of North America. Iowa, Kendall Hunt Publishing Company. p.687-722
Couto PHM, Araújo MS, Rodrigues PS, Della Lucia TMC, Oliveira MA \& Bacci L (2010). Formigas como bioindicadores da qualidade ambiental em diferentes sistemas de cultivo da soja. Revista Agrotecnologia, 1:11-20.

Delabie JHC, Paim VRLM, Nascimento IC, Campiolo S \& Mariano CSF (2006) As formigas como indicadores biológicos do impacto humano em manguezais da costa sudeste da Bahia. Neotropical Entomology, 35:602-615

Della Lucia TMC (2011) As formigas cortadeiras: da bioecologia ao manejo. Viçosa, Editora UFV. 421p.

Didham R (1997) The influence of edge effects and forest fragmentation on leaf litter invertebrates in central Amazonia. In: Laurance WF \& Bierregaard Junior RO (Eds.) Tropical forest remnants: ecology, management, and conservation of fragmented communities. Chicago, University of Chicago Press. p.55-70.

Didham RK (1998) Altered leaf-litter decomposition rates in topical forest fragments. Oecologia, 115:397-406.

Dobson A (2005) Monitoring global rates of biodiversity change: challenges that arise in meeting the Convention on Biological Diversity (CBD) 2010 goals. Philosophical Transactions of the Royal Society, 360:229-241.

Edge DA (2005) Butterfly conservation in the southern Cape. Metamorphosis, 16:28-46.

Engelbrecht IA (2010) Invertebrate species inventories in protected area management: are they useful? African Entomology, 18:235-245.

Fernández F \& Ospina M (2003) Sinopsis de las hormigas de la región Neotropical. In: Fernandéz F (Eds.) Introducción a las hormigas de la región Neotropical. Bogotá, Instituto de Investigación de Recursos Biológicos Alexander Von Humbolt. p.49-64.

Flather CH, Wilson KR, Dean DJ \& McComb WC (1997) Identifying gaps in conservation networks: of indicators and uncertainty in geographic-based analyses. Ecological Applications, 7:531-542.

Folgarait PJ (1998) Ant biodiversity and its relationship to ecosystem functioning, a review. Biodiversity and Conservation, 7:1221-1244.

Freitas AVL, Leal IR, Uehara-Prado M \& Iannuzzi L (2006) Insetos como indicadores de conservação da paisagem. In: Rocha CFD, Bergallo HG, Van Sluys M \& Alves MAS (Eds.) Biologia da Conservação. Rio de Janeiro, Editora da UERJ. p.201-225.

Frouz J (1999) Use of soil dwelling Diptera (Insecta, Diptera) as bioindicators: a review of ecological requirements and response to disturbance. Agriculture, Ecosystems \& Environment, 74:167-186.

Gadelha BQ, Ferraz ACP \& Coelho VMA (2009) A importância dos mesembrinelíneos (Diptera: Calliphoridae) e seu potencial como indicadores de preservação ambiental. Oecologia Brasiliensis, 13:661-665

Gardner TA, Barlow J, Araujo IS, Ávila-Pires TC, Bonaldo AB, Costa JE, Esposito MC, Ferreira LV, Hawes J, Hernandez MIM, Hoogmoed MS, Leite RN, Lo-Man-Hung NF, Malcolm JR, Martins MB, Mestre LAM, Miranda-Santos R, Overal WL, Parry L, Peters SL, Ribeiro-Junior MA, da Silva MNF, Motta CS \& Peres CA (2008) The costeffectiveness of biodiversity surveys in tropical forests. Ecological Letters, 11:139-150.

Gaston KJ \& Blackburn TM (1995) Mapping biodiversity using surrogates for species richness: macro-scales and New World birds. Proceedings of the Royal Society of London, 262:335-341.

Gorenflo LJ, Romaine S, Mittermeier RA \& Painemilla-Walker K (2012) Co-occurrence of linguistic and biological diversity in biodiversity hotspots and high biodiversity wilderness areas. Proccedings of the National Academy of Sciences, 109:8032-8037.

Hammer KC, Hill JK, Lace LA \& Langan AM (1997) Ecological and biogeographical effects of forest disturbance on tropical butterflies of Sumba, Indonesia. Journal of Biogeography, 24:67-75. 
Hammond PC \& Miller JC (1998) Comparison of the biodiversity of Lepidoptera within three forested ecosystems. Annals of the Entomological Society of America, 91:323-328.

Hellawell JM (1986) Biological Indicators of Freshwater Pollution and Environmental Management. Londres, Elsevier Applied Science. $559 \mathrm{p}$.

Hellawell JM (1991) Development of a rationale for monitoring. In: Goldsmith FB (Eds.) Monitoring for Conservation and Ecology. London, Chapman \& Hall. p.1-14.

Hill JK, Hammer KC, Lace LA \& Banham WMT (1995) Effects of selective logging on tropical forest butterflies on Buru, Indonesia. Journal of Applied Ecology, 32:754-760.

Hölldobler B \& Wilson EO (1990) The ants. Cambridge, Belknap. 732p.

Hutcheson J (1990) Characterization of terrestrial insect communities using quantified, Malaise-trapped Coleoptera. Ecological Entomology, 15:143-151.

Kemp M, Kock KN, Wepener V, Roets W, Quinn L \& Wolmarans CT (2014) Influence of selected abiotic factors on aquatic macroinvertebrate assemblages in the Olifants River catchment, Mpumalanga, South Africa. African Journal of Aquatic Science, 39:141-149.

King JAR, Andersen AN \& Cutter AD (1998) Ants as bioindicators of habitat disturbance: validation of the functional group model for Australia's humid tropics. Biodiversity and Conservation, 7:16271638

Kremen C (1992) Assessing the indicator properties of species assemblages for natural areas monitoring. Ecological Applications, 2:203-217.

LaSalle J \& Gauld ID (1993) Hymenoptera: their diversity, and their impact on the diversity of other organisms. In: Lasalle J \& Gauld ID (Eds.) Hymenoptera and Biodiversity. Wallingford, CAB International. p.1-26.

Leal IR, Filgueiras BKC, Gomes JP, Iannuzzi L \& Andersen AN (2012) Effects of habitat fragmentation on ant richness and functional composition in Brazilian Atlantic Forest. Biodiversity and Conservation, 21:1687-1701.

Levings SC (1983) Seasonal, annual and among site variation in the ground ant community of a deciduous tropical forest, some cause of patchy species distributions. Ecological Monographs, 53:435-455.

Louzada J \& Schiffler G (1996) Efeitos do fogo sobre a composicão e estrutura da comunidade de Scarabaeidae (Insecta: Coleoptera) na restinga da Ilha de Guriri, norte do ES. In: Miranda H, Saito C \& Dias B (Eds.) Impactos de queimadas em áreas de cerrado e restinga. Brasília, Editora UNB. p.161-169.

Louzada JNC \& Lopes FS (1997) A comunidade de Scarabaeidae copronecrófagos (Coleoptera) de um fragmento de Mata Atlântica. Revista Brasileira de Entomologia, 41:117-121.

Majer JD \& Kock AE (1992) Ant recolonization of sand mines near Richards Bay, South Africa: an evaluation of progress with rehabilitation. South African Journal of Science, 88:31-36.

Majer JD \& Nichols OG (1998) Long-term recolonization patterns of ants in Western Australian rehabilitated bauxite mines with reference to their use as indicators of restoration success. Journal of Applied Ecology, 35:161-182.

Majer JD (1983) Ants: bio-indicators of minesite rehabilitation, landuse, and land conservation. Environmental Management, 7:375-85.

Majer JD (1996) Ant recolonization of rehabilitated bauxite mines at Trombetas, Pará, Brazil. Journal of Tropical Ecology, 12:257-273.

Margules CR, Nicholls AO \& Pressey RL (1988) Selecting networks of reserves to maximize biological diversity. Biological Conservation, 43:63-76.
Marinho CGS, Zanetti R, Delabie JHC, Schlindwein MN \& Ramos LS (2002) Diversidade de Formigas (Hymenoptera: Formicidae) da Serapilheira em Eucaliptais (Myrtaceae) e Área de Cerrado de Minas Gerais. Neotropical Entomology, 31:187-195.

Marinoni RC \& Dutra RRC (1997) Famílias de Coleoptera capturadas com armadilha malaise em oito localidades do Estado do Paraná, Brasil. Diversidades alfa e beta. Revista Brasileira de Zoologia, 14:751770

Martins RP \& Antonini Y (2002) Comportamento de nidificação de abelhas solitárias no Brasil. In: $5^{\circ}$ Encontro Sobre Abelhas de Ribeirão Preto, Ribeirão Preto. Anais, Faculdade de Filosofia, Ciências e Letras de Ribeirão Preto-USP. p.58-64.

Mata RA, McGeoch MA \& Tidon R (2008) Drosophilid assemblages as a bioindicator system of human disturbance in the Brazilian Savanna. Biodiversity and Conservation, 17:2899-2916.

McGeoch MA, Sithole H, Samways MJ, Simaika JP, Pryke JS, Picker M, Uys C, Armstrong AJ, Dippenaar-Schoeman AS, Engelbrecht IA, Braschler B \& Hamer M (2011) Conservation and monitoring of invertebrates in terrestrial protected areas. Koedoe, 53:1-13.

Meffe GK \& Carrol CR (1997) Principles of Conservation Biology. $3^{\text {a ed. }}$ Massachussets, Sinauer. 699p.

Mendelik Y, Dayan T \& Feitelson E (2005) Planning for Biodiversity: the Role of Ecological Impact Assessment. Conservation Biology, 19:12541261.

Merrit RW, Cummins KW \& Berg MB (2008) An Introduction to the Aquatic Insects of North America. $4^{\mathrm{a} e d}$. Iowa, Kendall Hunt Publishing. 1214p.

Miranda PN, Morato EF, Oliveira MA \& Delabie JHC (2013) A riqueza e composição de formigas como indicadores dos efeitos do manejo florestal de baixo impacto em floresta tropical no Estado do Acre. Revista Árvore, 37:163-173.

Morato EF \& Campos LAO (2000) Efeitos da fragmentação florestal sobre vespas e abelhas solitárias em uma área da Amazônia Central. Revista Brasileira de Zoologia, 17:429-444.

Morato EF (2001) Biologia e ecologia de Anthodioctes moratoi Urban (Hymenoptera, Megachilidae, Anthidiini) em matas contínuas e fragmentos na Amazônia Central, Brasil. Revista Brasileira de Zoologia, 18:729-736.

Morrison ML (1986) Bird populations as indicators of environmental change. In: Johnston RF (Ed.) Current Ornithology. New York, Plenum. p.429-451.

Nichols E, Larsen T, Spector S, Davis AL, Escobar F, Favila M \& Vulinec $\mathrm{k}$ (2007) Global dung beetle response to tropical forest modification and fragmentation: A quantitative literature review and meta-analysis. Biological Conservation, 137:1-19.

Niemi G \& McDonald M (2004) Application of ecological indicators. Annual Review of Ecology, Evolution, and Systematics, 35:89-111.

Norris RH \& Thoms MC (1999) What is river health? Freshwater Biology, 41:197-209.

Numelin ML, Lodenius M, Tulisalo E, Hirvonen H \& Alanko T (2007) Predatory insects as bioindicators of heavy metal pollution. Environmental Pollution, 145:339-347.

Oliveira MA \& Della Lucia TMC (1992) Levantamento, de Formicidae de chão em áreas mineradas sob recuperação florestal de Porto Trombetas - Pará. Boletim do Museu Paraense Emílio Goeldi, 2:375-384.

Oliveira MA, Della Lucia TMC, Araujo MS \& Cruz AP(1995) A fauna de formigas em povoamentos de eucalipto e mata nativa no Estado do Amapá. Acta Amazônica, 25:117-126.

Oliveira MA, Della Lucia TMC, Marinho CGS, Delabie JHC \& Morato EF (2009) Ant diversity in an area of the amazon forest in Acre, Brazil. Sociobiology, 54:243-268. 
Oliveira MA, Della Lucia, TMC, Morato EF, Amaro MA \& Marinho CGS (2011) Vegetation structure and richness: effects on ant fauna of the Amazon - Acre, Brazil (Hymenoptera: Formicidae). Sociobiology, 57:243-267

Oliver I \& Beattie AJ (1996) Designing a cost-effective invertebrate survey: A test of methods for rapid assessment of biodiversity. Ecological Applications, 6:594-607.

Olivier L, Mélanie P, Sophie P, Chantal T, Michaëlle L, Frédéric D \& Hervé P (2012) Honey bees and pollen as sentinels for lead environmental contamination. Environmental Pollution, 170:254-259.

Otavo SE, Parrado-Rosselli A \& Noriega JA (2013) Superfamilia Scarabaeoidea (Insecta: Coleoptera) como elemento bioindicador de perturbación antropogénica en un parque nacional amazônico. Revista de Biología Tropical, 61:735-752.

Paoletti MG \& Bressan M (1996) Soil invertebrates as bioindicators of human disturbance. Critical Reviews in Plant Sciences, 15:21-62.

Pape T, Bickel D \& Rudolf M (2009) Diptera Diversity: Status, Challenges and Tools. Leiden, Brill Academic Publishers. 459p.

Pearce JL \& Venier LA (2006) The use of ground beetles (Coleoptera: Carabidae) and spiders (Araneae) as bioindicators of sustainable forest management: a review. Ecological Indicators, 6:780-793.

Peck SL, Mcquaid B \& Campbel CL (1998) Using ant species (Hymenoptera: Formicidae) as a biological indicator of agroecosystem condition. Environmental Entomology, 27:1102-1110.

Pereira JL, Picanço MC, da Silva, AA, Barros EC, Silva RS, Galdino TVS \& Marinho CGS (2010) Ants as environmental impact bioindicators from insecticide application on corn. Sociobiology, 54:153-164.

Price PW (1984) Insect Ecology. 2aed. New York, John Wiley \& Sons $607 \mathrm{p}$.

Rapport DJ (1992) Evolution of indicators of ecosystem health. In: McKenzie DH, Hyatt ED \& McDonald JV (Eds.) Ecological Indicators. New York, Elsevier Applied Science. p.121-134.

Resh VH \& Jackson JK (1993) Rapid assessment approaches to biomonitoring using benthic macroinvertebrates. In: Rosenberg DM \& Resh VH (Eds.) Freshwater Biomonitoring and Benthic Macroinvertebrates. New York, Chapman \& Hall. p.195 -233.

Ribas CR, Solar RRC, Campos RBF, Schmidt FA, Valentim CL \& Shoereder JH (2012) Can ants be used as indicators of environment impacts caused by arsenic? Journal of Insect Conservation, 16:413421.

Rizo-Patrón FV, Kumar A, Colton MBM, Springer M \& Trama FA (2013) Macroinvertebrate communities as bioindicators of water quality in conventional and organic irrigated rice fields in Guanacaste, Costa Rica. Ecological Indicators, 29: 68-78.
Rosenberg DM \& Resh VH (1993) Introduction to freshwater biomonitoring and benthic macroinvertebrates. In: Rosenberg DM \& Resh VH (Eds.) Freshwater biomonitoring and benthic macroinvertebrates. New York, Chapman and Hall. p.1-9.

Santos JC, Delabie JHC \& Fernandes GWA (2008) 15-year post evaluation of the fire effects on ant community in an area of Amazonian forest. Revista Brasileira de Entomologia, 52:82-87.

Silva RR \& Brandão CRF (1999) Formigas (Hymenoptera: Formicidae) como indicadores da qualidade ambiental e da biodiversidade de outros invertebrados terrestres. Biotemas, 12:55-73.

Silvestre R, Brandão CRF \& Silva RR (2003) Grupos funcionales de hormigas: el caso de lós grêmios Del Cerrado. In: Fernández F (Eds.) Introducción a las hormigas de la región Neotropical. Bogotá, Instituto de Investigación de Recursos Biológicos Alexander Von Humbolt. p.113-149.

Soares-Filho BS, Silvestrini R, Nepstad D, Brando P, Rodrigues H, Alencar A, Coe M, Locks C, Lima L \& Viana L (2012) Forest fragmentation, climate change and understory fire regimes on the Amazonian landscapes of the Xingu headwaters. Landscape Ecology, 27:585-599.

Spitzer K, Jaros J, Havelka J \& Leps J (1997) Effect of small-scale disturbance on butterfly communities of an Indochinese montane rainforest. Biological Conservation, 80:9-15.

Triplehorn CA \& Johnson NF (2011) Estudo dos Insetos. $7^{\mathrm{a}}$ ed. São Paulo, Cengage Learning. 816p.

Underwood EC \& Fisher BL (2006) The role of ants in conservation monitoring: If, when, and how. Biological Conservation, 132:166-182.

Uys C, Hamer M \& Slotow R (2006) The effect of burn area on invertebrate recolonisation in grasslands in the Drakensberg, South Africa. African Zoology, 41:51-65.

Vane-Wright RI, Smith CR \& Kitching IJ (1994) Systematic assessment of taxic diversity by summation. In: Forey PL, Humphries JC \& VaneWright RI (Eds.) Systematics and Conservation Evaluation. Oxford, Claredon Press. p.309-326.

Vasconcelos HL, Leite MF, Vilhena JMS, Lima PA \& Magnusson WE (2008) Ant diversity in an Amazonian savanna: Relationship with vegetation structure, disturbance by fire, and dominant ants. Austral Ecology, 33:221-231.

Weston DP \& Lydy ML (2014) Toxicity of the insecticide fipronil and Its degradates to benthic macroinvertebrates of urban streams. Environmental Science Technoogy, 48:1290-1297.

Winner RW, Boesel MW \& Farrell MP (1980) Insect community structure as an index of heavy-metal pollution in lotic ecosystems. Canadian Journal of Fisheries and Aquatic Science, 37:647-655.

Wood B \& Gillman MP (1998) The effects of disturbance on forest butterflies using two methods of sampling in Trinidad. Biodiversity and Conservation, 7:597-616. 